\title{
Comparison of Antioxidant Activity Ginseng (Panax Ginseng CA Meyer) Root Extraction between Ultrasound and Microwave Processing
}

\author{
Chengwen $\mathrm{Lu}^{1}$, Yongguang Yin ${ }^{2,}{ }^{*}$ \\ ${ }^{1}$ College of Life Science, Changchun Normal University, Changchun 130032, PR China \\ ${ }^{2}$ College of Biological and Agricultural Engineering, Jilin University, Changchun 130022, PR China \\ a biofoodyyg@hotmail.com
}

Keywords: ultrasound extraction, microwave extraction, ginsenoside, antioxidant activity.

\begin{abstract}
We compared antioxidant activity and biological compounds of ginseng root extractions between ultrasound (US) and microwave extraction (ME). The maximum total ginsenoside content of ginseng root extraction was $27.53 \mathrm{mg} / \mathrm{g}$ under the ultrasound conditions of $60^{\circ} \mathrm{C}$ for $2 \mathrm{~h}(50 \mathrm{kHz}$, powder $250 \mathrm{~W})$. It was higher than ME processing $(23.74 \mathrm{mg} / \mathrm{g})$ under the condition of powder $300 \mathrm{~W}$ for $8 \mathrm{~min}$. Moreover, the ginseng root extraction by US2 contained more total polyphenol $(11.51 \mathrm{mg} / \mathrm{g})$ and flavonoid $(13.37 \mathrm{mg} / \mathrm{g})$ than ME2 treatment. Moreover, the ginseng root extractions of UE treatment showed more powerful scavenging activities against DPPH, ABTS radicals and higher FRAP activity. Scanning electron microscopic (SEM) images of the ginseng root tissue with US and ME processing provided visual evidence of the disruption effect. The US procedure used for ginseng root processing contributed to enhance extraction efficiencies and antioxidant activity of functional materials.
\end{abstract}

\section{Introduction}

Panax ginseng C.A. Meyer (ginseng), a well-known natural medicine, has been frequently used in Asian countries as a traditional medicine. More than 60 kinds of ginsenosides, described chemically as ginseng saponins, have been isolated and pharmacological characterized (Cheng et al. 2008). The aglcone moieties of these saponins were steroids or complex triterpenoids, which were linked to sugar molecules. The extractions of Panax ginseng contained a broad range of common ginsenosides including ginsenosides $\mathrm{Re}, \mathrm{Rd}, \mathrm{Rb}$ and $\mathrm{Rf}$, etc. and rare ginsenosides such as ginsenosides $\operatorname{Rg} 3, \operatorname{Rg} 5$, Rs3 and Rh3. Depend on the type of skeletons and sugar moieties, ginsenosides have various biological functions, including anticancer, anti-cardiovascular, antioxidant and mental capacity improvement (Zhao et al. 2013; Chen et al. 2016; Shergis et al. 2013).

It has been reported different methods of ginsenoside extraction, including heat reflux extraction (Gafner et al. 2004), hydrolytic enzymes extraction (Lee et al. 2012), carbon dioxide extraction (Wang et al. 2001) and ultrahigh pressure extraction (Chen et al. 2009). Ultrasound has proven to be a much simpler and more effective processing than the traditional extraction method for extracting ginseng saponins from various ginseng roots. The ultrasound-assisted saponins extraction from ginseng roots was three times faster than the conventional thermal extraction (Wu et al. 2001). Microwave-assisted technique has been used to extract ginsenosides Rg1 and Rb1 from ginseng root under atmospheric pressure. Microwave-assisted extraction $\operatorname{Rg} 1$ (15min) were better than convertional solvent extraction (10h) (Shu et al. 2003). High pressure microwave assisted extraction (HPMAE) was applied to extract the ginsenosides from Panax gingseng root. The results indicated that the HPMAE not only took a short time but also afforded higher extraction yields of ginsenosides (Wang et al. 2008).

The objective of this study was to compare the total ginsenoside content, antioxidant activity and biological compounds under US and ME extraction. In this paper, we reported our experimental method and results. The results might provide useful information for ginsenoside extraction from Panax ginseng roots. 


\section{Materials and Methods}

\subsection{Materials}

Ginseng roots (four year old) were obtained from Jilin province, dried in vacuum at $60^{\circ} \mathrm{C}$ until a constant weight, and passed through an 80 mesh screen. HPLC analytical grade solvents were purchased from J.T. Baker, USA. All other chemical reagents were analytical grade and deionized water was used to prepare sample solutions.

\subsection{Instruments and Apparatus}

Centrifuge biofuge heraeus (4000-40,000 r/min); Magnetic stirrer; The HPLC instrument (Agilent 1100, HP Technologies, USA); Ultrasonic cell grinder (JY96-II); microwave oven (Media 800W microwave output powder, $2450 \mathrm{MHz}$ ).

\subsection{Methods of Extracting Ginsenosides From Ginseng Root}

\subsubsection{Ultrasound Treatment}

The dried ginseng powder (1g) was mixed with 50mL 70\% ethanol solution, and then treated with ultrasonic cell grinder on the condition of $60^{\circ} \mathrm{C}(50 \mathrm{kHz}, 250 \mathrm{~W}$ heat power) for $1 \mathrm{~h}$ (US1), $2 \mathrm{~h}$ (US2) and $3 \mathrm{~h}$ (US3).

\subsubsection{Microwave Treatment}

The dried ginseng powder (1g) was mixed with $50 \mathrm{~mL} 70 \%$ ethanol solution, and then treated on the condition of powder $300 \mathrm{~W}$ for $6 \mathrm{~min}$ (ME1), $8 \mathrm{~min}$ (ME2) and 10min (ME3).

\subsection{Determination of Total Ginsenoside Content}

The HPLC analysis was performed using Agilent 1100 system with a reverse phase $\mathrm{C}_{18}$ column $(4.6 \times 150,5 \mu \mathrm{m})$ and a UV spectrophotometric detector. The column temperature was maintained at $30^{\circ} \mathrm{C}$. The mobile phase consisted of solvent A (water) and solvent B (acetonitrile) were used for separation. The solvent gradient condition was $20 \%$ B $(0-20 \mathrm{~min}), 20 \% \mathrm{~B}(20-31 \mathrm{~min}), 32 \% \mathrm{~B}$ (31-40min), 43\% B (40-70min), 100\% B (70-80min) at a flow rate of $1 \mathrm{~mL} / \mathrm{min}$. The eluate was measured at a wavelength of $203 \mathrm{~nm}$ and the injection volume was $10 \mu \mathrm{L}$. The retention times of ginsenoside standards were used to identify chromatographic peaks.

\subsection{Determination of Total Polyphenol Content}

Total polyphenol content of extractions were determined according to the method of Singleton and Lamuela-Raventos (1999) with minor modification. One milliliter of each extraction and 1.0mL of diluted Folin-Ciocalteu reagent were mixed. After $5 \mathrm{~min}$ incubation, $1.0 \mathrm{~mL}$ of $10 \%$ sodium carbonate was added and the mixture was incubated for $1 \mathrm{~h}$. The absorbance at $760 \mathrm{~nm}$ was measured and converted to polyphenol content according to the calibration curve of gallic acid.

\subsection{Determination of Total Flavonoid Content}

Total flavonoid content was determined by using a colorimetric method described previously (Woisky and Salatino, 1998). Dried solvent extracts $(0.2 \mathrm{~g})$ were dissolved by $80 \%$ methanol $20 \mathrm{~mL}$, extracted for $2 \mathrm{~h}$ at room temperature and centrifuged at $5000 \mathrm{~g}$ for $20 \mathrm{~min}$ (Jung et al. 2006). Then $80 \%$ methanol was added to the extract until $100 \mathrm{~mL}$ volume. The $\mathrm{AlCl}_{3}$ ethanol solution $(2 \%)$ was added to $0.5 \mathrm{~mL}$ extract. After reaction $1 \mathrm{~h}$ at room temperature, the absorbance was measured at $420 \mathrm{~nm}$.

\subsection{DPPH Radical Scavenging Activity}

The DPPH radical scavenging activity was determined using the method described by Yang et al. (2006). The DPPH power $(2.5 \mathrm{mg})$ was dissolved in $10 \mathrm{~mL}$ ethanol and mixed completely. DPPH solution in ethanol $(2 \mathrm{~mL})$ was added to $2 \mathrm{~mL}$ sample solution. The absorbance of the extraction was measured at $514 \mathrm{~nm}$ at room temperature, using ethanol as the blank.

$$
S A(\%)=1-\frac{A_{I}-A_{J}}{A_{0}} \times 100
$$

$A_{i}=$ absorbance of $2 \mathrm{~mL}$ DPPH $+2 \mathrm{~mL}$ sample; $A_{j}=$ absorbance of $2 \mathrm{~mL}$ sample $+2 \mathrm{~mL}$ ethanol; $A_{0}=$ absorbance of $2 \mathrm{~mL}$ DPPH $+2 \mathrm{~mL}$ ethanol. 


\subsection{ABTS Radical Scavenging Activity}

The ABTS antioxidant activity was carried out using the method of $\mathrm{Hu}$ and Kitts (2001). The ABTS radical cation was prepared by mixing $7 \mathrm{mM}$ ABTS stock solution with $2.45 \mathrm{mM}$ potassium persulfate, and kept in darkness for 14h. The absorbance of ABTS solution was adjusted to 0.7 with distilled water. The ginsenoside root extraction $50 \mu \mathrm{L}$ was added to $2 \mathrm{~mL}$ ABTS radical solution for reacting $6 \mathrm{~min}$. The spectrophotometer was used to measure the ABTS antioxidant activity at $734 \mathrm{~nm}$ using trolox as positive control. The inhibition ratio (\%) was calculated using the following formula:

Inhibition ratio $(\%)=\left(\mathrm{A}_{0}-\mathrm{A}_{1}\right) / \mathrm{A}_{0} \times 100$

where $A_{0}$ is the absorbance of the control, and $A_{1}$ is the absorbance of the test sample.

\subsection{FRAP Assay}

The FRAP assay was performed following the method described by Chen et al. (2010). The ginseng root extraction was mixed with the FRAP solution for $1 \mathrm{~h}$ in dark condition. The absorbance was determined at $593 \mathrm{~nm}$ against water as a blank. The results were expressed in $\mu$ mol Trolox equivalents (TE)/g FW.

\subsection{Analysis Structure Changes of Ginseng Root Tissue After Extraction}

To elucidate the effect of each extraction procedure on structure changes, ginseng root extraction with different processing were analyzed by scanning electron microscopy (SEM). The sample particles were fixed on a specimen holder with aluminum tape and then sputter-coated with gold. The samples were examined with a SSX-550 (Shimadzu, Japan) SEM under high vacuum condition (Chen et al., 2009).

\subsection{Statistical Analysis}

Analysis of one way ANOVA and Duncan's multiple range tests were performed by SPSS software (Version 13.0). All the experiments were completed in three times and all data were presented as standard deviations (SD). For each analysis, a level of significance 5\% was considered significant.

\section{Results and Discussion}

\subsection{Comparison Ginsenoside Content Between US and ME Extraction}

We performed parallel experiments using the same raw material from the same batch. Total ginsenoside content from ginseng root treated by US and ME methods were shown in Fig.1. With the same ginseng root, the US method produced higher yield of total saponin than ME method. Under the US condition of $60^{\circ} \mathrm{C}$ for $2 \mathrm{~h}$, the yield of total saponin achieved maximum $27.53 \mathrm{mg} / \mathrm{g}$. Its content was 1.40 times higher than ME1 treatment for $6 \mathrm{~min}, 1.16$ times higher than ME2 treatment for $8 \mathrm{~min}$. The results indicated that the extractability of total saponin increased during US and ME processing. Therefore, the US and ME treatment were promising methods to obtain active ingredients.

\subsection{Comparison Total Polyphenol Content Between US and ME Extraction}

Total polyphenol compounds were the secondary metabolites synthesized in the plant, and current researches indicated that dietary phenolic compound had antioxidative, anti-inflammatory and anticarcinogenic activities (Yang et al., 2001). Under the US2 processing condition of $60^{\circ} \mathrm{C}$ for $2 \mathrm{~h}$, the content of total polyphenol achieved maximum $11.51 \mathrm{mg} / \mathrm{g}$, which is $60.98 \%$ higher compared to the ME1 treatment for $6 \mathrm{~min}$ and $14.07 \%$ higher compared to the ME2 treatment for $8 \mathrm{~min}$ (Fig.2). The accumulation of total phenolic compounds may have an impact on the biological activities of ginseng root extraction (Kim et al., 2011).

\subsection{Comparison Total Flavonoid Content Between US and ME Extraction}

More efficient antioxidant activity in ginseng root extraction originates from the presence of higher concentrations of such potent antioxidant compounds. Flavonoid is one of the most powerful antioxidants in plants (Rice-Evans et al., 1996). Total flavonoid contents of ginseng root extraction were summarized in Fig. 3. The US treatment contained higher content of total flavonoid $(13.37 \mathrm{mg} / \mathrm{g})$ than ME1 treatment for $6 \mathrm{~min}(10.41 \mathrm{mg} / \mathrm{g})$ and ME2 treatment for $8 \mathrm{~min}(12.13 \mathrm{mg} / \mathrm{g})$. 


\subsection{Comparison DPPH Radical Scavenging Activity Between US and ME Extraction}

The structure of ginsenoside and its antioxidant activities have an interdependent relationship (Liu et al., 2003). Therefore, it is necessary to compare the biological activity of ginseng root extraction by US and ME methods. Fig. 4 showed the DPPH radical scavenging activity of ginseng root extraction with different methods. Among the different methods, ginseng root extraction of US2 processing $2 \mathrm{~h}$ (48.31\%) seemed relatively effective in scavenging activity, followed by US3 treatment for $3 \mathrm{~h}$ $(45.46 \%)$, ME2 treatment for $8 \min (44.23 \%)$ and US1 treatment for $1 \mathrm{~h}(43.2 \%)$. Therefore, the extractions of US processing showed significant anti-radical activity, as measured by their capacity to scavenge the stable free radical DPPH.

\subsection{Comparison ABTS Radical Scavenging Activity Between US and ME Extraction}

The antioxidant activity as well as free radical scavenging effects of ginseng root has been extensively investigated. ABTS radical scavenging was used to measure the antioxidant capacity of ginseng based on its ability to reduce radical cations (Kang et al., 2007). In the present study, the results of ABTS radical scavenging activity were shown in Fig. 5. The US2 treatment (2h) showed significantly higher ABTS radical scavenging activity (41.04\%) compared to the ME1 treatment for $6 \min (34.35 \%)$ and ME2 treatment for $8 \min (38.16 \%)$. From these results, it confirmed that the ginseng extraction of US processing had greater antioxidant capacity.

\subsection{Comparison FRAP Activity Between US and ME Extraction}

Antioxidant capacity of ginseng root extraction as evaluated by FRAP was summarized in Fig. 6. The ginseng root extraction by US processing showed higher FRAP values than ME processing. The FRAP values of US treatment showed increasing tendency until the condition of $60^{\circ} \mathrm{C}$ for $2 \mathrm{~h}$, then decreased with processing time. The FRAP values achieved $5.27 \mu \mathrm{mol}$ (TE)/g FW at the conditions of $60{ }^{\circ} \mathrm{C}$ with ultrasonic cell grinder $(50 \mathrm{kHz}, 250 \mathrm{~W}$ heat power) for $2 \mathrm{~h}$. The analysis clearly demonstrated that US processing was capable of improving the antioxidant capacity of ginseng root extraction.

\subsection{Comparison Structure Changes of Ginseng Particles Between US and ME Extraction}

The samples treated with US and ME processing were taken for scanning electron microscopy (SEM) analysis. The sectioned particles were fixed on a specimen holder with aluminum tape and then sputtered with a thin coating of gold. The different extraction methods produced distinguishable physical changes in ginseng root. Fig. 7a showed the SEM micrograph of the untreated samples, the cells of the ginseng roots tissues were kept intact. In the case of ME2 treatment, puny damage was observed of treated samples (Fig. 7b). The hollow openings were generated and smaller particles were developed with the US2 treatment (Fig. 7c). The results indicated that the cell walls of the plant tissues were broken and all the cell constituent components were washed away by US and ME processing. These results indicated that US processing induced a subsequent change in the surface tension of the cellulose, and a number of small particles appeared of ginseng root tissues.

\section{Conclusion}

In this study, we carried out to investigate the total ginsenoside content and antioxidant activity of ginseng root extraction by US and ME processing. Compared with the ME methods, the US treatment provided high extraction efficiency. Moreover, the US treatment contained more total polyphenol and flavonoid content, higher DPPH radical scavenging activity, ABTS radical scavenging activity and FRAP activity compared with ME treatment. When choosing an extraction method, one should consider both extraction efficiency and the maximum antioxidant activity. Ultrasound extraction was a feasible alternative method for extracting interested ingredients from biological materials. 


\section{Acknowledgements}

This study was supported by National Natural Science Foundation of China (31601400) and Natural Science Foundation from Jilin Province Department of Science and Technology (20140101040JC).

\section{References}

[1]. Chen, C.O., Ribaya-Mercado, J.D., Mckay, D.L., Croom, E. and Blumberg, J.B. 2010. Differential antioxidant and quinone reductase inducing activity of American, Asian, and Siberian ginseng. Food Chem. 119, 445-451.

[2]. Chen, R.Z., Meng, F.L., Zhang, S.Q. and Liu, Z.Q. 2009. Effects of ultrahigh pressure extraction conditions on yields and antioxidant activity of ginsenoside from ginseng. Sep. Purif. Technol., 66: $340-346$.

[3]. Chen, Y., Liu, Z.H., Xia, J., Li, X.P., Li, K.Q., Xiong, W., ...Chen, D.L. 2016. 20(S)-ginsenoside $\mathrm{Rh} 2$ inhibits the proliferation and induces the apoptosis of $\mathrm{KG}-1 \mathrm{a}$ cells through the Wnt/ $\beta$-catenin signaling pathway. Oncol. Rep. 36, 137-146.

[4]. Cheng, L.Q., Na, J.R., Bang, M.H., Kim, M.K. and Yang, D.C. 2008. Conversion of major ginsenoside Rb1 to 20(S)-ginsenoside Rg3 by Microbacterium sp. GS514. Phytochemistry, 69: 218-224.

[5]. Gafner, S., Bergeron, C., Mccollom, M.M., Cooper, L.M., Mcphall, K.L., Gerwick, W.H. and Angerhofer, C.K. 2004. Evaluation of the efficiency of three different solvent systems to extract triterpene saponins from roots of Panax quinquefolius using high-performance liquid chromatography. J. Agr. Food Chem. 52, 1546-1550.

[6]. Hu, C. and Kitts, D.D. 2001. Free radical scavenging capacity as related to antioxidant activity and ginsenoside composition of Asian and North American ginseng extracts. J. Am. Oil. Chem. Soc., 78: 249-255.

[7]. Jung, C.H., Seog, H.M., Choi, I.W., Park, M.W. and Cho, H.Y. 2006. Antioxidant properties of various solvent extracts from wild ginseng leaves. LWT-Food Sci. Technol., 39: 266-274.

[8]. Kang, K.S., Yamabe, N., Kim, H.Y., Okamoto, T., Sei, Y. and Yokozawa, T. 2007. Increase in the free radical scavenging activities of American ginseng by heat processing and its safety evaluation. J. Ethnopharmacol., 113: 225-232.

[9]. Kim, B.G., Choi, S.Y., Suh H.J. and Park, H.J. 2011. Bitterness reduction and enzymatic transformation of ginsenosides from Korean red ginseng (Panax ginseng) extract. J. Food Biochem., 35: 267-1282.

[10]. Lee, H.J., Lee, H.S., Cho, H.J., Sun, Y.K. and Suh, H.J. 2012. Utilization of hydrolytic enzymes for the extraction of ginsenosides from korean ginseng leaves. Process Biochem. 47, 538-543.

[11]. Liu, Z.Q., Luo, X.Y., Liu, G.Z., Chen, Y.P., Wang Z.C. and Sun, Y.X. 2003. In vitro study of the relationship between the structure of ginsenoside and its antioxidative or prooxidative activity in free radical induced hemolysis of human erythrocytes. J .Agr. Food Chem., 51: 2555-2558.

[12]. Rice-Evans, C.A., Miller N.J. and Pagana, G. 1996. Structure- antioxidant activity relationship of flavonoids and phenolic acids. Free Radical Bio. Med., 20: 933-956.

[13]. Shergis, J.L., Zhang, A.L., Zhou, W. and Xue, C.C. 2013. Panax ginseng in randomised controlled trials: a systematic review. Phytother. Res. 27, 949-965. 
[14]. Shu, Y.Y., Ko M.Y. and Chang, Y.S. 2003. Microwave-assisted extraction of ginsenosides from ginseng root. Microchem. J., 74: 131-139.

[15]. Singleton, V.L. and Lamuela-Raventos, R.M. 1999. Analysis of total phenols and other oxidation substrates and antioxidants by means of Folin-Ciocalteu reagent. Method. Enzymol., 299: $152-178$.

[16]. Wang, H.C., Chen, C.R. and Chang, C.J. 2001. Carbon dioxide extraction of ginseng root hair oil and ginsenosides. Food Chem. 72, 505-509.

[17]. Wang, Y.T., You, J.Y., Yu,Y., Qu, C.L., Zhang, H.R., Ding L., et al., 2008. Analysis of gingsenosides in Panax ginseng in high pressure microwave-assisted extraction. Food Chem., 110: $161-167$.

[18]. Woisky, R.G. and Salatino, A. 1998. Analysis of propolis: Some parameters and procedures for chemical quality control. J. Agri. Res., 37: 99-105.

[19]. Wu, J., Lin L. and Chau, F. 2001. Ultrasound-assisted extraction of ginseng saponins from ginseng roots and cultured ginseng cells. Ultrason. Sonochem., 8: 347-352.

[20]. Yang, B., Wang, J.S., Zhao, M.M., Liu, Y., Wang, W. and Jiang, Y.M. 2006. Identification of polysaccharides from pericarp tissues of litchi (Litchi chinensis Sonn.) fruit in relation to their antioxidant activities. Carbohyd. Res., 341: 634-638 .

[21]. Yang, C.S., Landau, J.M. Huang, M.T. andNewmark, H.L. 2001. Inhibition of carcinogenesis by dietary polyphenolic compounds. Annu. Rev. Nutr., 21:3 81-406.

[22]. Zhao, L.G., Xie, J.C., Zhang, X.S., Cao, F.L. and Pei, J.J. 2013. Overexpression and characterization of a glucose-tolerant $\beta$-glucosidase from Thermotoga thermarum DSM 5069T with high catalytic efficiency of ginsenoside Rb1 to Rd. J. Mol. Catal. B-Enzym. 95, 62-69. 